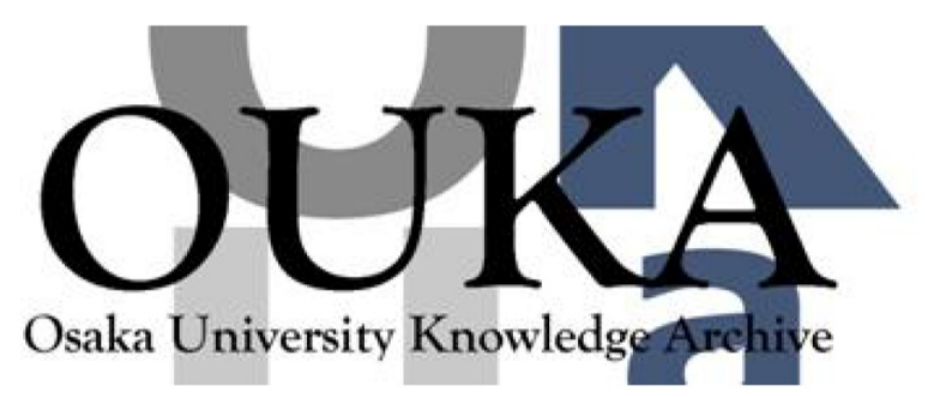

\begin{tabular}{|c|c|}
\hline Title & $\begin{array}{l}\text { Spin-fluctuation-mediated even-parity pairing } \\
\text { in heavy-fermion superconductors }\end{array}$ \\
\hline Author (s) & Miyake, K. ; Schmitt-Rink, S.; Varma, C. M. \\
\hline Citation & Physical Review B. 34(9) p. 6554-p. 6556 \\
\hline Issue Date & $1986-11-01$ \\
\hline oaire:version & VoR \\
\hline URL & https://hdl. handle. net/11094/3161 \\
\hline rights & $\begin{array}{l}\text { Miyake, K., Schmitt-Rink, S., Varma, C. M., } \\
\text { Physical Review B, 34, 9, 6554-6556, 1986-11- } \\
\text { 01. "Copyright } 1986 \text { by the American Physical } \\
\text { Society." }\end{array}$ \\
\hline Note & \\
\hline
\end{tabular}

Osaka University Knowledge Archive : OUKA

https://ir. Library. osaka-u. ac. jp/

0saka University 


\title{
Spin-fluctuation-mediated even-parity pairing in heavy-fermion superconductors
}

\author{
K. Miyake, ${ }^{*}$ S. Schmitt-Rink, and C. M. Varma \\ AT\&T Bell Laboratories, Murray Hill, New Jersey 07974 \\ (Received 27 June 1986; revised manuscript received 11 August 1986)
}

\begin{abstract}
It is shown that the anisotropic even-parity pairings are assisted and the odd-parity as well as the isotropic even-parity pairings are impeded by antiferromagnetic spin fluctuations which are observed in heavy-fermion solids.
\end{abstract}

The nature of the attractive interaction and the type of Cooper pairing in heavy-fermion superconductors have led to a lively discussion. ${ }^{1}$ There are at least three points of views:

(a) It is conventional phonon-mediated pairing driven by the electron-phonon interaction via the strong volume dependence of the Kondo temperature. ${ }^{2,3}$

(b) Since the Kondo effect leads to a strong on-site repulsion in the singlet channel, the pair wave function has a node at the origin, but near-neighbor electron-electron attraction in the singlet channel via exchange of phonons produces pairing in an anisotropic singlet state. ${ }^{4-6}$

(c) Because of the same reason as in (b) the pair wave function must have a node at the origin. Further, the heavy-fermion phenomena are completely dominated by spin fluctuations, ${ }^{7}$ and it is the exchange of these that must be the microscopic cause of pairing. ${ }^{7,8}$ At this point, this reasoning is developed ${ }^{8,9}$ on the basis of a vague expectation that the physics must be similar to that in the case of superfluidity of liquid ${ }^{3} \mathrm{He}$ where ferromagnetic spin fluctuations (FMSF) lead to triplet pairing. ${ }^{10-14}$

Our basic physical ideas here are the same as in (c) above, but we study the nature of pairing due to antiferromagnetic spin fluctuations (AFMSF) since such fluctuations are experimentally observed. ${ }^{15-18} \mathrm{We}$ find, as is commonly believed, that AFMSF suppress conventional singlet pairing; they also suppress triplet pairing but promote anisotropic singlet pairing. The conclusion that they suppress triplet pairing was arrived at by Matsuura et al. ${ }^{5}$ by Hirsch ${ }^{19}$ and by Béal-Monod, Bourbonnais, and Emery. ${ }^{20}$ A suggestion that AFMSF promotes anisotropic singlet pairing is contained in the work of Hirsch. ${ }^{19}$

A strong motivation for our investigation is the experimental results which indicate that heavy-fermion superconductors do indeed have pairing of an unusual kind with most likey line or lines of zeros of the gap function on the Fermi surface. $^{21}$ Group theoretical investigations have revealed that such a gap function cannot occur in the triplet odd-parity manifold in the presence of strong spin-orbit scattering, ${ }^{22-26}$ but can occur in the singlet even-parity manifold. ${ }^{23,25}$ A complete classification of such states is given by Volovik and Gorkov. ${ }^{23}$

The main assumptions made in this paper are as follows: (a) The frequency dependence of the spin-fluctuationmediated interaction is neglected as in Refs. 11 and 12 for the ${ }^{3} \mathrm{He}$ problem. (b) The repulsive interaction $I$ among heavy-fermion quasiparticles is assumed to be momentum independent, so that the spin or pseudospin (of quasiparti- cles) is conserved. Then the effective interaction Hamiltonian close to a magnetic instability is ${ }^{11,12}$

$$
\begin{aligned}
& H_{\text {int }}=\frac{1}{2} \sum_{k k^{\prime}} \sum_{q} J\left(k-k^{\prime}\right) \sigma_{\alpha \beta} \cdot \sigma_{\gamma \delta} a_{k}^{+}+q / 2, \alpha \\
& \times a_{-k+q / 2, \gamma}^{+} a_{-k^{\prime}+q / 2, \delta} a_{k^{\prime}+q / 2, \beta} .
\end{aligned}
$$

$J(q)$ can be expresed in terms of $\chi(q)$, the $q$-dependent susceptibility, using the random-phase approximation, $J(q)=-(I / 2)[1+I \chi(q)]$. The validity of such approximations is doubtful. However, given an experimental $\chi(q)$, one can be reasonably certain that the $q$ dependence of $J(q)$ is similar to it: For ferromagnetic fluctuations $J(q)$ has its maximum at $q=0$, and for antiferromagnetic fluctuations at some finite wave vector.

First we start with the unphysical but instructive example where $J(q)$ is isotropic and as in Fig. 1. The curves 1 and 2 represent the ferromagnetic case (1) and antiferromagnetic case (2), respectively. In the weak coupling approximation, i.e., $T_{c} \ll T_{F}$, the heavy-fermion bandwidth, the partial-wave components of the pairing interaction $V_{l}$ are given by

$$
V_{l}=\left\{\begin{array}{r}
3 \\
-1
\end{array}\right\} 2 \int_{0}^{1} d x x P_{l}\left(1-2 x^{2}\right)\left[-J\left(2 k_{F} x\right)\right],
$$

where the upper (lower) case corresponds to spin singlet (triplet) and even (odd) parity.

If $J\left(2 k_{F} x\right)$ is independent of $x, V_{l}>0$ for the spin singlet $l=0$ case and $V_{l}=0$ for all $l \neq 0$. This is easy to under-

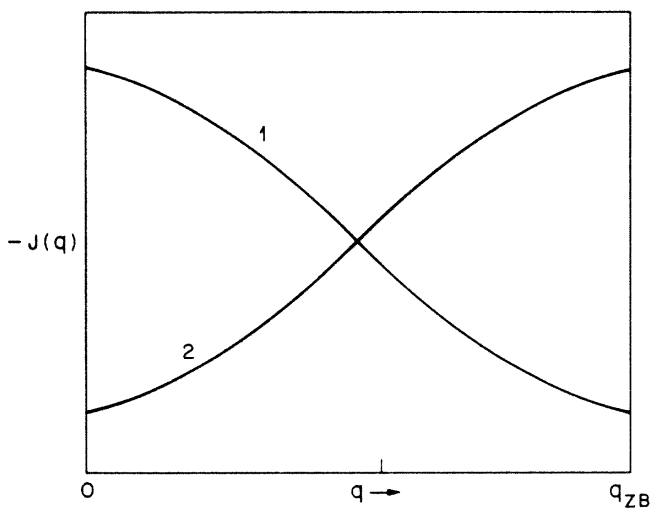

FIG. 1. Effective exchange interaction $J(q)$ as a function of $q$ for ferromagnetic (1) and antiferromagnetic (2) spin fluctuations. 
stand. $J(q)$ independent of $q$ means a delta-function interaction in real space where any finite $l$ Cooper pair wave function has zero magnitude. For $l=0$ a constant $J(q)$ has the same effect as a magnetic impurity, i.e., it suppresses pairing.

It is easy to see, from (2) and the monotonic variation in curves (1) and (2) of Fig. 1, that $V_{0}>0$ and $V_{1}<0$ in case (1) in agreement with the case of ${ }^{3} \mathrm{He}$, whereas $V_{0}>0$ and $V_{1}>0$ in the case (2), i.e., antiferromagnetic spin fluctuations impede both the singlet $s$-wave and the triplet $p$-wave pairing. The sign of $V_{l}(l>2)$ depends on the details of the form of $J(q)$. This example shows that the effect of AFMSF on the pairing interaction is quite different from that of FMSF.

Next we examine a more realistic model where the crystal symmetry is taken into account. It is a common feature for $\mathrm{CeCu}_{2} \mathrm{Si}_{2}$ (Ref. 15), $\mathrm{UBe}_{13}$ (Refs. 15 and 16), and $\mathrm{UPt}_{3}$ (Refs. 15, 17, and 18) that the AFMSF are well developed. In fact, for $\mathrm{UBe}_{13}$ and $\mathrm{UPt}_{3}$ a small perturbation, such as replacing magnetic ions by nonmagnetic ones, makes the system magnetically ordered. Though we have no detailed knowledge about the structure of spin fluctuations, it may be reasonable to assume that the spin susceptibility $\chi(q)$ and so $-J(q)$ have a maximum at some point $q=Q$ along one of the symmetry axes of the Brillouin zone. Therefore, we may approximate $-J(q)$ near its maximum as

$$
-J(q)=J_{0}-J_{1} \gamma_{q},
$$

where the positive constants $J_{0}$ and $J_{1}$ are $O\left(T_{F}\right)$, and $\gamma_{q}$ is a function which has a minimum at some point along a symmetry axis. The pairing potential $V_{k-k^{\prime}}$ is expressible in the form

$$
V_{k-k^{\prime}}=\left\{\begin{array}{r}
3 \\
-1
\end{array}\right\}\left(J_{0}-J_{1} \gamma_{k-k^{\prime}}\right) \text {. }
$$

We examine two cases as nontrivial examples: (i) $Q=(\pi / a, \pi / a, \pi / a)$ and its equivalents, corresponding to an antiferromagnetic SDW with a fcc sublattice in a sc lattice $(a \times a \times a)$. (ii) $Q=(\pi / a, \pi / a, 0)$ and its equivalents, corresponding to an antiferromagnetic array in (110) direction of ferromagnetic sheets in a fcc lattice $(2 a \times 2 a \times 2 a)$.

Case (i): The function $\gamma_{q}$, which has a minimum at $Q=(\pi / a, \pi / a, \pi / a)$, may be represented as $\gamma_{q}=2\left(C_{q}^{x}\right.$ $\left.+C_{q}^{y}+C_{q}^{z}\right)$, with the abbreviations $C_{q}^{x} \equiv \cos \left(q_{x} a\right)$ and $S_{q}^{y} \equiv \sin \left(q_{y} a\right)$, etc. One can see by elementary algebra that the pairing interaction $V_{k-k},(4)$, can be written in a separable form ${ }^{4,5}$

$$
\begin{aligned}
& V_{k-k^{\prime}}^{\text {even }}=3 J_{0}-\frac{J_{1}}{2}\left(\phi_{k}^{(1)} \phi_{k^{\prime}}^{(1)}+\eta_{k}^{(1)} \eta_{k^{\prime}}^{(1)}+\zeta_{k}^{(1)} \zeta_{k^{\prime}}^{(1)}\right), \\
& V_{k-k^{\prime}}^{\text {odd }}=2 J_{1} \sum_{i=x, y, z} S_{k}^{i} S_{k^{\prime}}^{i},
\end{aligned}
$$

where $\phi_{k}^{(1)}=\gamma_{k}, \quad \eta_{k}^{(1)}=\sqrt{6}\left(C_{k}^{x}-C_{k}^{y^{\prime}}\right), \quad \zeta_{k}^{(1)}=\sqrt{2}\left(C_{k}^{x}+C_{k}^{y}\right.$ $\left.-2 C_{k}^{z}\right)$. The functions $\phi_{k}^{(1)}, \eta_{k}^{(1)}, \zeta_{k}^{(1)}$, and $S_{k}^{i}(i=x, y, z)$ are mutually orthogonal in the sense $\sum_{k} \phi_{k}^{(1)} \eta_{k}^{(1)} \times$ (function with full symmetry of the crystal) $=0$, etc., and $\phi_{k}^{(1)}$, $\eta_{k}^{(1)}$ and $\zeta_{k}^{(1)}$, and $S_{k}$ have $s$-like, $d \gamma$-like, and $p$-like symmetry, respectively. Notice that the spin-fluctuationmediated interactions for the triplet odd-parity pairing, $2 J_{1} \Sigma_{i} S_{k}^{i} S_{k}^{i}$, and the singlet isotropic pairing, $3 J_{0}$, are repulsive, while those for the anisotropic even-parity pairing, $-\frac{1}{2} J_{1}\left(\phi_{k}^{(1)} \phi_{k}^{(1)}+\eta_{k}^{(1)} \eta_{k}^{(1)}+\zeta_{k}^{(1)} \zeta_{k}^{(1)}\right)$, are attractive. This feature holds also for other cases and crystal symmetries. The gap equation for the even-parity pairing is written as

$$
\begin{aligned}
& \Delta_{k}=\sum_{k^{\prime}} {\left[-3 J_{0}+\frac{1}{2} J_{1}\left(\phi_{k}^{(1)} \phi_{k^{\prime}}^{(1)}+\eta_{k}^{(1)} \eta_{k^{\prime}}^{(1)}+\zeta_{k}^{(1)} \zeta_{k^{\prime}}^{(1)}\right)\right] } \\
& \times \frac{\Delta_{k^{\prime}}}{2 E_{k^{\prime}}} \tanh \left(\frac{E_{k^{\prime}}}{2 T}\right),
\end{aligned}
$$

where $E_{k}=\left(\xi_{k}^{2}+\left|\Delta_{k}\right|^{2}\right)^{1 / 2}, \xi_{k}$ being the kinetic energy of the heavy-fermion quasiparticles. Because of the orthogonality among $\left(1, \phi_{k}^{(1)}, \eta_{k}^{(1)}, \zeta_{k}^{(1)}\right)$, the gap function $\Delta_{k}$ can be expressed as

$$
\Delta_{k}=\Delta_{1}+\Delta_{\phi} \phi_{k}^{(1)}+\Delta_{\eta} \eta_{k}^{(1)}+\Delta_{\zeta} \zeta_{k}^{(1)}
$$

It is easy to see that the transition temperatures $T_{c}^{d \gamma}$ for the $\eta$ and $\zeta$ components are degenerate and that $T_{c}^{\phi}$ for the $\phi$ component is different in general. In order to determine which $T_{c}$ is higher and what type of gap structure develops below $T_{c}$, we need more knowledge about the form of $\xi_{k}$. However, one can see from the discussions in Ref. 4 that (a) if $T_{c}^{d \gamma}<T_{c}^{\phi}$, then the stable gap function is $\Delta_{k}=\Delta_{1}+\Delta_{\phi} \phi_{k}^{(1)}$ which has the full symmetry of the crystal, and (b) if $T_{c}^{d \gamma}>T_{c}^{\phi}$, then $\Delta_{k}=\Delta\left(\eta_{k}^{(1)} \pm i \zeta_{k}^{(1)}\right)+$ (small admixture of 1 and $\left.\phi_{k}^{(1)}\right)$. The gap in case (a) corresponds to the identical (one-dimensional) representation $A_{1 \mathrm{~g}}$ of class $O \times R$ in Volovik-Gorkov ${ }^{23}$ (VG) but it can vanish along lines on the Fermi surface (FS) ${ }^{5}$ as pointed out by Ohkawa and Fukuyama, ${ }^{6}$ because the two surfaces $\xi_{k}=0$ and $\Delta_{k}=0$ can intersect for a wide range of parameters of the model. The gap in case (b) corresponds to the twodimensional representation $E_{g}$ of class $O\left(D_{2}\right)$ in VG and it vanishes at points of intersection of the FS with the threefold axis.

Case (ii): The function, which has a minimum at $Q=(\pi / a, \pi / a, 0)$, is given by

$$
\gamma_{q}=4\left(C_{q}^{x} C_{q}^{y}+C_{q}^{y} C_{q}^{z}+C_{q}^{z} C_{q}^{x}\right)+2 \beta\left(C_{q}^{x}+C_{q}^{y}+C_{q}^{z}\right),
$$

with $\beta<4$. The pairing interaction $V_{k}-k^{\prime}$ can be expressed in a way similar to (5) with additional basis functions such as

$$
\begin{aligned}
& \phi_{k}^{(2)}=\sqrt{8}\left(C_{k}^{x} C_{k}^{y}+C_{k}^{y} C_{k}^{z}+C_{k}^{z} C_{k}^{x}\right), \\
& \eta_{k}^{(2)}=\sqrt{12}\left(C_{k}^{x}-C_{k}^{z}\right) C_{k}^{y}, \zeta_{k}^{(2)}=2\left(C_{k}^{x} C_{k}^{y}+C_{k}^{y} C_{k}^{z}-2 C_{k}^{z} C_{k}^{x}\right), \\
& \lambda_{k}=\sqrt{24} S_{k}^{x} S_{k}^{y}, \mu_{k}=\sqrt{24} S_{k}^{y} S_{k}^{z}, v_{k}=\sqrt{24} S_{k}^{z} S_{k}^{x},
\end{aligned}
$$

and so on:

$$
\begin{aligned}
& V_{k-k^{\prime}}^{\text {even }}=3 J_{0}-\frac{1}{2} J_{1}\left(\phi_{k}^{(2)} \phi_{k^{\prime}}^{(2)}+\eta_{k}^{(2)} \eta_{k^{\prime}}^{(2)}+\zeta_{k}^{(2)} \zeta_{k^{\prime}}^{(2)}+\lambda_{k} \lambda_{k^{\prime}}+\mu_{k} \mu_{k^{\prime}}+v_{k} v_{k^{\prime}}\right)-\frac{\beta}{2} J_{1}\left[\phi_{k}^{(1)} \phi_{k^{\prime}}^{(1)}+\eta_{k}^{(1)} \eta_{k^{\prime}}^{(1)}+\zeta_{k}^{(1)} \zeta_{k^{\prime}}^{(1)}\right] \\
& V_{k-k^{\prime}}^{\text {odd }}=4 J_{1}\left[C_{k}^{x} S_{k}^{y} C_{k}^{x} S_{k}^{y,}+C_{k}^{y} S_{k}^{x} C_{k}^{y \prime} S_{k}^{x,}+\left(\begin{array}{l}
\text { cyclic exchange } \\
\text { of } x, y, z
\end{array}\right)\right]+2 \beta J_{1} \sum_{i=x, y, z} S_{k}^{i} S_{k^{\prime}}^{i} .
\end{aligned}
$$


Again, the spin-fluctuation-mediated pairing interactions for the odd-parity pairing and isotropic even-parity pairing are repulsive, and those for anisotropic even-parity pairing are attractive. In principle, all types of even-parity pairing except the three-dimensional representation $F_{1 g}$ in VG are possible for the interaction (8), including the fully symmetric gap function of the Ohkawa-Fukuyama type, which vanishes on lines on the FS. To determine which type of pairing is realized in this manifold, we need again more detailed knowledge about the parameters $\beta, J_{0}$, and $J_{1}$, and the form of $\xi_{k}$.

One can do similar calculations for other types of crystal symmetry such as tetragonal and hexagonal. For reasonable forms of the function $\gamma_{q}$ the qualitative results given above remain valid. ${ }^{27}$
In this paper, we have tried to reconcile the physical idea that the heavy-fermion behavior is dominated by spin fluctuations with the observation of superconductivity with (most likely) a gap vanishing along lines on the Fermi surface. Such a gap is forbidden in the triplet odd-parity manifold but allowed in the singlet even-parity manifold. We have looked at a few simple possible dispersions of antiferromagnetic spin fluctuations and found that such superconducting states are preferred. ${ }^{27}$ More theoretical work in this direction must await "a more complete determination of the spin-fluctuation dispersion by neutron scattering.

We would like to thank G. Aeppli, B. Batlogg, and D. J. Bishop for discussion of experimental results.
${ }^{*}$ Permanent address: Department of Physics, Nagoya University, Chikusa-Ku, Nagoya 464, Japan.

${ }^{1}$ C. M. Varma, Comments Solid State Phys. 11, 221 (1985); P. A. Lee, T. M. Rice, J. W. Serene, L. J. Smith, and J. W. Wilkins, ibid. 12, 99 (1986).

${ }^{2}$ H. Razafimandimby, P. Fulde, and J. Keller, Z. Phys. B 54, 111 (1984).

${ }^{3}$ R. M. Martin and J. W. Allen, J. Magn. Magn. Mater. 31-34, 473 (1983).

${ }^{4}$ K. Miyake, T. Matsuura, H. Jichu, and Y. Nagaoka, Prog. Theor. Phys. 72, 1063 (1984).

${ }^{5}$ T. Matsuura, K. Miyake, H. Jichu, Y. Kuroda, and Y. Nagaoka, J. Magn. Magn. Mater. 52, 239 (1985).

${ }^{6}$ F. J. Ohkawa and H. Fukuyama, J. Phys. Soc. Jpn. 53, 4344 (1984)

${ }^{7}$ C. M. Varma, Bull. Am. Phys. Soc. 29, 404 (1984); Phys. Rev. Lett. 55, 2723 (1985).

${ }^{8}$ P. W. Anderson, Phys. Rev. B 30, 1549 (1984).

${ }^{9}$ O. T. Valls and Z. Tesanovic, Phys. Rev. Lett. 53, 1497 (1984).

${ }^{10}$ A. Layzer and D. Fay, Int. J. Magn. 1, 135 (1971).

${ }^{11}$ P. W. Anderson and W. F. Brinkman, Phys. Rev. Lett. 30 , 1108 (1973)

${ }^{12}$ S. Nakajima, Prog. Theor. Phys. 50, 1101 (1973).

${ }^{13}$ Y. Kuroda, Prog. Theor. Phys. 51, 1269 (1974); 53, 349 (1975).

${ }^{14}$ W. F. Brinkman, J. W. Serene, and P. W. Anderson, Phys. Rev. A 10, 2386 (1974).

${ }^{15}$ For references of experiments, see, e.g., the following review papers: G. R. Stewart, Rev. Mod. Phys. 56, 755 (1984); F. Steglich, in Theory of Heavy Fermions and Valence Fluctuations, edited by K. Kasuya and T. Saso, Springer Series in
Solid State Sciences, Vol. 62 (Springer, New York, 1985), p. 23.

${ }^{16}$ B. Batlogg, D. Bishop, B. Golding, C. M. Varma, Z. Fisk, J. L. Smith, and H. R. Ott, Phys. Rev. Lett. 55, 1319 (1985).

${ }^{17}$ G. Aeppli, A. Goldman, G. Shirane, E. Bucher, and M.-Ch. Lux-Steiner (unpublished).

${ }^{18}$ A. P. Ramirez, B. Batlogg, A. S. Cooper, and E. Bucher, Bull. Am. Phys. Soc. 31, 446 (1986); and (unpublished).

${ }^{19}$ J. E. Hirsch, Phys. Rev. Lett. 54, 1317 (1985). A related idea was presented by V. J. Emery, Synth. Met. 13, 21 (1986).

${ }^{20}$ M. T. Béal-Monod, C. Bourbonnais, and V. J. Emery (unpublished).

${ }^{21}$ S. Schmitt-Rink, K. Miyake, and C. M. Varma (unpublished); P. Hirschfeld, D. Vollhardt, and P. Wölfle (unpublished), also arrived at a similar conclusion.

22P. W. Anderson, Phys. Rev. B 30, 4000 (1984).

${ }^{23}$ G. E. Volovik and L. P. Gorkov, Pis'ma Zh. Eksp. Teor. Fiz. 39, 550 (1984) [JETP Lett. 39, 674 (1984)]; Zh. Eksp. Teor. Fiz. 88, 1412 (1985) [Sov. Phys. JETP 61, 843 (1985)].

${ }^{24}$ K. Ueda and T. M. Rice, Phys. Rev. B 31, 7114 (1985).

${ }^{25}$ E. I. Blount, Phys. Rev. B 32, 2935 (1985).

${ }^{26}$ Classification of odd-parity pairing without spin-orbit coupling has been done by M. Ozaki, K. Machida, and T. Ohmi, Prog. Theor. Phys. 74, 221 (1985); 75, 442 (1986).

${ }^{27}$ However, the possibility of the following complicated case cannot be excluded: If $\gamma_{q}$ in (3) is given by $\gamma_{q}=C_{q}^{x}+C_{q}^{y}-C_{q}^{z}$ for tetragonal symmetry, the pairing interaction can be attractive also for the odd-parity pairing with the $S_{k}^{z}$ component. Such a $\gamma_{q}$ corresponds to AFMSF in (110) direction and FMSF in (001) direction. 